\title{
Technical note: Assessing lameness in tie-stalls using live stall lameness scoring
}

\author{
S. Palacio, ${ }^{11}$ L. Peignier,† C. Pachoud,ł C. Nash,§ S. Adam,\# R. Bergeron,§ D. Pellerin,\|l A. M. de Passillé,ף \\ J. Rushen,ף D. Haley, ${ }^{* *}$ T. J. DeVries, $\S$ and E. Vasseur* \\ *Animal Science, McGill University, Ste-Anne-de-Bellevue, Quebec, H9X 3V9, Canada \\ †AgroCampus Ouest, 35000 Rennes, France \\ ¥Montpellier SupAgro, 34060 Montpellier, France \\ §Animal Biosciences, University of Guelph, Guelph, Ontario, N1G 2W1, Canada \\ \#Valacta, Ste-Anne-de-Bellevue, Quebec, H9X 3R4, Canada \\ IIDépartement des Sciences Animales, Université Laval, Quebec, Quebec, G1V 0A6, Canada \\ TDairy Education and Research Centre, University of British Columbia, Agassiz, British Columbia, V0M 1A1, Canada \\ ${ }^{* *}$ Population Medicine, University of Guelph, Guelph, Ontario, N1G 2W1, Canada
}

\section{ABSTRACT}

Video stall lameness scoring (SLS) has been shown to be comparable to video locomotion scoring for evaluating lameness in dairy cows housed in tie-stalls and may be a more practical and easier method to measure lameness in a herd. We compared live SLS to video SLS and to live locomotion scoring. A total of 685 lactating cows subsampled from 27 commercial dairy herds were examined for lameness through live and video SLS. Cows scored with the live or video SLS system were scored for 4 behavioral indicators while still in their stall: weight shifting (shift), standing on the edge of the stall (edge), uneven weight bearing while standing (rest), and uneven weight bearing while moving from side to side (uneven). Two observers live scored and video scored for SLS. Lameness prevalence from video SLS and live SLS were similar (31 vs. 30\%, respectively). Prevalence of the behavioral indicators varied from 0.59 to $58.2 \%$. Sensitivity and specificity of live SLS was calculated using video SLS as the gold standard for lameness detection in tie-stalls. Sensitivity of live SLS was 0.83 and specificity was 0.94 . False positives and false negatives for lameness were 14.4 and $16.8 \%$, respectively. When comparing the prevalence of lameness measured through video or live SLS at the herd level, live SLS for lameness was correlated to video SLS $(\mathrm{r}=0.91)$ with a Cohen's kappa coefficient of 0.79 (95\% confidence limit $=0.73-0.84$ ). Average exact agreement in the behavior indicators observed ranged from 80 to $100 \%$. A subsample of 250 cows from 5 herds were scored for live SLS and live locomotion by a third observer. Intra- and interobserver reliability

Received October 17, 2016.

Accepted April 19, 2017.

${ }^{1}$ Corresponding author: spalacio89@gmail.com for live SLS and live locomotion scoring were found to have a kappa coefficient of 0.53 (95\% confidence limit $=0.43-0.64)$ when determining a cow as lame through SLS or locomotion scoring. Live SLS was correlated with live locomotion scoring $(\mathrm{r}=0.92)$. However, lameness prevalence was lower when using live SLS (28.4\%) compared with locomotion scoring (38\%). In summary, live SLS may be an acceptable method to replace video SLS to identify lame cows and rank tie-stall herds in terms of lameness prevalence without having to remove the cows from their stalls or view videos to score; however, it may underestimate lameness prevalence compared with locomotion scoring methods.

Key words: lameness, stall lameness scoring, dairy cow, tie-stall

\section{Technical Note}

Easy and reliable welfare measures are important for the future of the dairy industry as dairy producers are being required to provide assurances regarding animal welfare (Rushen et al., 2011; Barkema et al., 2015) to build public trust. Lameness is widely regarded as a major welfare issue in the dairy industry in all housing and milking systems and assessment of lameness prevalence is an important aspect of animal welfare assessment (e.g., Whay et al., 2003; Vasseur et al., 2015). In Canada, lameness prevalence has recently been recorded to be $21 \%$ for cows in free-stalls with a milking parlor (Solano et al., 2015), 15\% for cows in farms with automated milking systems (Westin et al., 2016), and $24 \%$ for cows in tie-stalls (Charlton et al., 2016). An accurate and easy method to score lameness in tie-stalls is needed as producers with tie-stall barns tend to put less effort into lameness control and monitoring (Higginson Cutler et al., 2015) and tie-stall barns represent 50\% of US dairy farms (USDA, 2010) and $71 \%$ of Cana- 
dian dairy farms (Canadian Dairy Information Centre, 2015). The stall lameness scoring (SLS) method was developed by Leach et al. (2009) as a means of detecting lame cows in their stalls, and SLS using video was later validated against locomotion scoring using video by Gibbons et al. (2014), which showed that SLS is an adequate method to score lameness in the stall. Locomotion scoring requires long corridors free of obstacles to reliably observe and score the gait of the cows; these are areas most tie-stall barns do not have. Live SLS does not require such areas and, additionally, does not require more time and extra equipment to record and score lameness, unlike video SLS. Consequently, live SLS may be more practical and less time consuming for producers, veterinarians, and field advisors. However, validating live SLS against video SLS is necessary before it can be used with confidence. Our objectives were to compare live SLS to (1) the previously validated video-based method of SLS and to (2) live locomotion scoring.

To address our first objective, a study was conducted in which a total of 685 lactating Holstein cows on 27 commercial tie-stall herds (between 14 and 40 cows per herd) in Ontario and Quebec, Canada, were observed. All methods used to collect data were approved by the University of Guelph's Animal Care Committee. Cows were selected as part of 2 other studies, where selection was based on DIM and parity, as one of the key outcome measures was lying time, which has been shown to be affected by DIM and parity (Vasseur et al., 2012). Each cow was video recorded and live SLS scores were taken, using the method adapted from Leach et al. (2009) and described in Gibbons et al. (2014) while the cow was in the stall. First, any lying cows were encouraged to stand by an experienced cow handler, who stood near the cow and vocally encouraged her to get up. If the cow did not respond, she was lightly tapped on the spine until she began to rise. If this was not enough, the cow was tapped on the flank and near the spine to encourage rising. If the cow did not rise, a new attempt was made at a later time until the cow had been assessed. Each cow was required to be standing undisturbed for a minimum of 3 min before the rest of the SLS protocol was followed. Adjacent cows were not filmed/scored immediately after each other, to prevent the disturbance of filming a cow from affecting the filming/scoring of the neighboring cows. The protocol was performed by 2 individuals: an experienced cow handler who controlled the camera and handled the cow and 1 trained observer who performed live scoring of the cow. The cow was filmed with a camcorder placed on a tripod. First, the cow's hind legs were filmed for 10 $\mathrm{s}$ with the camera about $1 \mathrm{~m}$ directly behind the cow.
Then, the camera was moved to the left of the cow to film a side view for $10 \mathrm{~s}$, followed by the same step performed to the right of the cow. The camera was then returned to the position directly behind the cow and the cow handler encouraged the cow to step from side to side 2 to 3 times per side. To do this, the cow handler first walked to one side of the cow in an attempt to encourage the cow to move in the opposite direction. The cow handler then repeated this in the opposite direction. If the cow did not respond to the handler's movements, the movement was repeated while the handler tapped gently around the cow's pin bone or flank on the side opposite to the direction the cow was being encouraged to move. If this did not work, the cow was gently prodded using a pen or finger on the pin bone or by quickly and lightly tapping the hoof on the opposite side to which the cow was required to move. This process of encouraging the cow to move was repeated a minimum of 4 times, and as many times as the live observer needed. During this process the observer stayed next to the camera to ensure a similar viewing angle between the camera and the observer. If at any point the cow started to urinate or defecate, the recording was stopped and scoring was attempted later to prevent the stance taken during these behaviors from potentially affecting the observations.

Video analysis was performed by the same observer who did the live scoring. Videos were scored between 2 and 6 wk after live scoring/recording. In an attempt to reduce scoring bias, the videos were randomized and no more than 4 videos from a single farm were scored sequentially. A total of 685 videos were scored by 2 observers ( $\mathrm{n}=196$ for observer 1 and $\mathrm{n}=489$ for observer 2).

The SLS method focuses on hind limbs and targets 4 behavioral indicators. Each of the 4 behavioral indicators observed are described by Gibbons et al. (2014) and assessed on whether they were present or not: (1) weight shift: regular, repeated shifting of weight from one hoof to another, defined as lifting each hind hoof completely off the ground at least twice. The hoof had to be lifted and returned to the same location; stepping forward or backward did not define this indicator. (2) Stand on edge: the cow places one or both of her hooves on the edge of the stall while standing stationary. This did not include times when both hind hooves were in the gutter or when the cow briefly placed her hoof on the edge during a movement or step. (3) Uneven weight: repeatedly resting one foot more than the other, indicated by the cow raising a part or the entire hoof off the ground. This did not include raising of the hoof to lick or during kicking. (4) Uneven movement: uneven weight bearing between the left and right feet when the 
cow was encouraged to move from side to side. This was demonstrated by a more rapid movement by one foot than the other or by an evident reluctance to bear weight on a particular foot. Following recommendations of Leach et al. (2009), we used as in Gibbons et al. (2014) 2 indicators to classify a cow as lame when using SLS, to decrease the number of possible false positives.

Both observers involved in the study were trained, and both intra- and interobserver reliability were assessed using videos established as standards by Gibbons et al. (2014), as well as 20 randomly selected videos from the first 60 video recordings from the present study. The percent agreement for intra- and interobserver reliability was calculated as number of exact agreements/ total number of observations $\times 100$. Cohen's kappa coefficients were calculated using SAS version 9.2 (SAS Institute Inc., Cary, NC) to assess agreement not due to chance. Kappa values for intra-observer reliability were $0.44(95 \%$ confidence limit $=-0.2-1.0$; exact agreement $\geq 90 \%)$ for edge, $0.78(95 \%$ confidence limit $=$ $0.38-1.0$; exact agreement $\geq 97 \%)$ for weight shift, 0.68 $(95 \%$ confidence limit $=0.45-0.91 ;$ exact agreement $\geq 84 \%$ ) for uneven weight, 0.53 (95\% confidence limit $=0.26-0.8$; exact agreement $\geq 76 \%)$ for uneven movement, and 0.64 (95\% confidence limit $=0.37-0.9$; exact agreement $\geq 84 \%$ ) for determining that a cow was lame when 2 or more behaviors were observed. These results are similar to those found by Gibbons et al. (2014) except for uneven movement, in which they observed higher intraobserver reliability (92\%). Kappa values for interobserver reliability were 0.48 (95\% confidence limit $=-0.12-1.0$; exact agreement $\geq 94 \%)$ for edge, 0.64 (95\% confidence limit $=0.19-1.0$; exact agreement $\geq 94 \%)$ for weight shift, $0.22(95 \%$ confidence limit $=$ $-0.19-0.64$; exact agreement $\geq 65 \%$ ) for uneven weight, $0.68(95 \%$ confidence limit $=0.36-1.0$; exact agreement $\geq 84 \%$ ) for uneven movement, and 0.20 (95\% confidence limit $=-0.27-0.67$; exact agreement $\geq 70 \%$ ) for determining a cow as lame when 2 or more behaviors were observed. These results are similar to those found by Gibbons et al. (2014); however, they observed higher interobserver exact agreement in uneven weight (95\%) and lameness when using $\geq 2$ behaviors as determinants (89\%).

Prevalence of behavior indicators based on video scoring was found to be lowest for edge and shift, and highest for rest and uneven (Table 1); this is in accordance with the data gathered by Gibbons et al. (2014; data not reported) in which they found the prevalence for edge $(14.4 \%)$ and shift $(1.1 \%)$ to be lower than rest $(57.4 \%)$ and uneven $(51.4 \%)$. The prevalence of lameness based on video was $31.0 \%$. The contribution of each behavior to a cow being determined as lame was lowest for edge and shift and highest for rest and uneven (Table 1). Although uneven contributes to $96.6 \%$ of the cows determined lame, it is important to note that others (Gibbons et al., 2014) have found that using 2 behaviors to determine cows as lame helps diminish the potential for false positives. Additionally, uneven is the only behavior that requires movement from the cows, making it a complex behavior to observe and perform; because of this, having the requirement of an additional behavior to confirm lameness makes the method more robust.

The sensitivity (proportion of true positives correctly identified) and specificity (proportion of true negatives correctly identified) of the live scoring method at detecting the presence of each behavior compared with the video method (gold standard) of scoring is shown in Table 1. Percentage of false positives and false negatives was highest for shift $(25.0 \%)$. This may be due to the fact that only 4 recorded cases of shift occurred in our study, meaning that every incorrectly matched observation accounted for $25.0 \%$ of the number labeled as false

Table 1. Prevalence of 4 behavioral indicators, and of lameness, observed with video scoring (gold standard) and live scoring of 685 cows in tie-stalls, across 27 herds, with test characteristics for live scoring ${ }^{1}$

\begin{tabular}{lccccc}
\hline Item & Edge & Shift & Rest & Uneven & Lameness \\
\hline \% Prevalence (video) & 7.9 & 0.59 & 39.3 & 58.2 & 30.8 \\
\% Prevalence (live) & 9.36 & 0.58 & 37.7 & 54.0 & 30.1 \\
\% Contribution $^{2}$ & 20.2 & 1.9 & 90.4 & 96.6 & \\
\% Only behavior observed $^{3}$ & 1.61 & 0 & 10.74 & 28.04 & \\
Sensitivity $_{\text {Specificity }}$ & 0.96 & 0.75 & 0.87 & 0.81 & 0.83 \\
\% False positives & 0.98 & 1.00 & 0.94 & 0.84 & 0.94 \\
\% False negatives & 22.2 & 25.0 & 8.6 & 11.3 & 14.4 \\
\hline
\end{tabular}

${ }^{1}$ For each method, lameness was defined by the presence of at least 2 indicators.

$2 \%$ contribution refers to percentage of cows defined as lame in which the indicator was observed when using video scoring.

$3 \%$ only behavior observed refers to percentage of cows observed to display only that behavior (using videos to score). 
positives or false negatives. However, even when prevalence for the indicators edge and shift was low, it was observed that sensitivity and specificity were still high (above $75.0 \%$ ), meaning that accuracy was still high, despite a single false positive or false negative having a larger effect on the accuracy of the live SLS. This low observance of shift may indicate that this behavior may not be a vital component of the SLS method and future studies may not require its inclusion.

The sensitivity and specificity for rest, edge, and uneven were above $80 \%$ with the sensitivity of uneven being the lowest at $0.81(81 \%)$ with video as the gold standard. A possible reason for the lower sensitivity for uneven could be because video observation allows observers to re-watch individual side-to-side movements more than one time, which might allow the observer to more easily pick up subtle abnormalities in the movement and perhaps make them more likely to interpret a movement as an indication of lameness. This means that when scoring uneven movement live, observers might underestimate presence of the behavior compared with video scoring. The prevalence observed for uneven movement supports this assumption because prevalence of uneven movement was $58.2 \%$ when scored through video and $54.0 \%$ when scored live. With regard to lameness, live SLS had a sensitivity and specificity of (0.83) 83 and (0.94) 94\%, respectively, when compared with video SLS. The high specificity indicates that when live scoring a cow as lame, the probability of the cow being scored as lame on video was high; however, live scoring may slightly underestimate lameness compared with video scoring, as indicated by the lower sensitivity rate.

Using SAS version 9.2 (SAS Institute Inc.) the herd-level correlations (Pearson correlation) as well as Cohen's kappa coefficients, controlling for the variance between herds by including the herd variable in the Cohen's kappa formula ahead of the video and live comparison were calculated comparing live SLS against video SLS for the 27 herds. Kappa coefficients and Pearson correlation coefficients were 0.81 (95\% confidence limit $=0.7-0.91 ;$ correlation $=0.93 ; P<0.001)$ for edge, Kappa not calculated due to low number of positive occurrences (correlation $=0.81 ; P<0.001$ ) for shift, $0.84(95 \%$ confidence limit $=0.79-0.88$; correlation $=0.90 ; P<0.001)$ for rest, 0.65 (95\% confidence limit $=0.59-0.71 ;$ correlation $=0.87 ; P<0.001)$ for uneven, and 0.79 (95\% confidence limit $=0.73-0.84$; correlation $=0.91 ; P<0.001)$ for lame, demonstrating that live scoring was comparable to video scoring. The indicator with the lowest correlation was shift. Again this is most likely due to the fact that prevalence of shift observed in both video or live scoring was very low, causing any difference in observation between the 2 methods to have a large effect on their correlation.

When comparing exact agreement of live SLS against video SLS for each indicator, we found the agreement between the methods to be $97.8 \%$ for edge, $99.7 \%$ for shift, $91.5 \%$ for rest, $81.2 \%$ for uneven, and $90.3 \%$ for lameness. Agreement of the indicators varied from 73.7 to $100 \%$ for edge, shift, and rest for all 27 herds when observed at the individual farm level. For uneven, the percentage of exact agreement ranged from 70.0 to $100 \%$ for 24 out of the 27 herds, whereas 3 herds had a percentage agreement for uneven of 50.0, 55.6, and $65.0 \%$. Exact agreement for lameness varied from 79.0 to $100 \%$ between all 27 herds. Exact agreement was lowest for the uneven weight bearing. This is similar to results reported by Gibbons et al. (2014), who found that uneven weight bearing was the least reliable indicator. This is most likely due to the fact that uneven weight bearing is a motion-sensitive indicator that, unlike the other indicators, is affected by the ease of manipulating the cow during the test, making it a more difficult indicator to assess properly.

To address our second objective, a second study was conducted using 250 lactating Holstein cows on 5 Ontario herds with sample sizes per herd varying from 31 to 59 animals. Each cow was live scored in the morning in their stall with SLS using the same procedural methods and training as Gibbons et al. (2014). The cows were later randomly removed from their stalls one at a time by the producer and locomotion scored by the observer in the afternoon of the same day. Locomotion scoring was adapted for on-farm use from Flower and Weary (2009) and a cow was categorized as lame when reluctant to bear weight on at least one limb (i.e., walked with a limp; Gibbons et al., 2014; Solano et al., 2015; Westin et al., 2016). Both live SLS and locomotion scorings were performed by the same observer (observer 3) for all cows. The observer trained with the same videos used to train in objective 1 and by Gibbons et al. (2014). Kappa values of inter- and intraobserver reliability, using the same standard videos established in Gibbons et al. (2014), were found to be 0.45 (95\% confidence limit $=0.04-0.86$; exact agreement above $73.0 \%$ ) when determining a cow as lame with both live SLS and locomotion scoring. Overall prevalence of lameness on the 250 cows observed using live SLS was $28.4 \%$ compared with the $38.0 \%$ observed with live locomotion scoring (Table 2). This difference of $9.6 \%$ between the 2 methods may be due to the high number of false negatives observed (41.1\%); a similar underestimation of lameness by live SLS compared with live locomotion was also observed by Leach et al. (2009). The Kappa coefficient and Pearson correlation adjusting for the dif- 
ferent herds between live SLS and live locomotion scoring at the herd level was $0.53(95 \%$ confidence limit $=$ $0.43-0.64$; correlation $=0.92 ; P<0.01)$. These results are similar to those found by Gibbons et al. (2014) with a correlation of $0.88(P=0.002)$ and may indicate that although SLS may underestimate lameness compared with locomotion scoring, it can still properly rank the farms with regard to lameness prevalence, making it a useful tool for categorizing herds. Sensitivity and specificity measured at the cow level (Table 2) indicate that using live SLS might underestimate the prevalence of lameness, but it is accurately determining true negative cases of lameness. This is in accordance with the results found by both Leach et al. (2009) and Gibbons et al. (2014) where the sensitivity of the SLS was lower than its specificity when compared with locomotion scoring. Exact agreement at the cow level when comparing live SLS to live locomotion scoring was $78.4 \%$. Because currently no other tools are available to assess lameness on tied cows and producer accuracy in detecting lameness has been reported to be around 20 to $30 \%$ (Whay et al., 2003; Espejo et al., 2006), these results suggest that live SLS may be an acceptable method to score lameness in tie-stalls without the need to move tied cows out of their stalls when locomotion scoring is not an option.

Overall, our results suggest that live SLS is a comparable method to video SLS to score and identify lame cows in tie-stall herds, as it is in agreement with the previously validated video SLS method. A substantial agreement was found between live SLS and video SLS when classifying a cow as lame, even when some behaviors proved to be more difficult to score (uneven). When comparing live SLS with live locomotion scoring, live SLS seems to underestimate lameness and provide some false negatives. This may indicate that live SLS may not be the strongest method in identifying lameness at the cow level. However, live SLS and live locomotion have a strong linear correlation at the herd level, suggesting that live SLS may prove useful in ranking and categorizing herds with respect to lameness prevalence, especially if locomotion scoring is not possible. Our results show that although there was a low specificity, most likely due to the low repeatability of the observer, live SLS can still accurately rank farms in terms of lameness prevalence. Therefore, live SLS is an adequate and simple method to assess lameness in tie-stalls compared with video SLS as it does not require extra time to observe videos or extra equipment to score. It has the potential to provide producers and advisers with a way to rank and estimate herd lameness prevalence without requiring the cows to be removed from their stalls. However, one must consider that SLS may underestimate lameness compared with locomotion scoring.
Table 2. Prevalence of lameness using locomotion scoring (gold standard) and live stall lameness scoring (SLS), with test characteristics for $\operatorname{SLS}^{1}$

\begin{tabular}{lcc}
\hline Item & Lameness & $\begin{array}{c}\text { Kappa } \\
\text { coefficient }\end{array}$ \\
\hline \% Prevalence (locomotion) & 38 & $0.53^{2}$ \\
Prevalence (SLS) & 28.4 & \\
Sensitivity & 0.59 & \\
Specificity & 0.9 & \\
\% False positives & 9.68 & \\
\% False negatives & 41.1 & \\
\hline
\end{tabular}

${ }^{1}$ For SLS, lameness was defined as the presence of at least 2 behavioral indicators. Observations on 250 cows across 5 herds.

${ }^{2}$ Kappa coefficient for agreement between locomotion scoring and SLS.

\section{ACKNOWLEDGMENTS}

We thank the participating farmers from Quebec and Ontario, Canada. This study was funded by the Dairy Farmers of Canada (Ottawa, Ontario, Canada), the Canadian Dairy Network (Guelph, Ontario, Canada), the Canadian Dairy Commission (Ottawa, Ontario, Canada), Agriculture and Agri-Food Canada (Ottawa, Ontario, Canada), as part of the Dairy Science Cluster 2 and Organic Science Cluster II initiatives, supported by the AgriInnovation Program of Agriculture and Agri-Food Canada's Growing Forward 2 Policy Framework (a federal-provincial-territorial initiative). We thank Roger Cue (McGill University, Montreal, Quebec, Canada) for providing consultation with the statistical analyses.

\section{REFERENCES}

Barkema, H. W., M. A. G. von Keyserlingk, J. P. Kastelic, T. J. G. M. Lam, C. Luby, J.-P. Roy, S. J. LeBlanc, G. P. Keefe, and D. F. Kelton. 2015. Invited review: Changes in the dairy industry affecting dairy cattle health and welfare. J. Dairy Sci. 98:7426-7445.

Canadian Dairy Information Centre. 2015. The farm: Dairy barns by type in Canada. Accessed Aug. 9, 2016. http://www.dairyinfo. gc.ca/index_e.php?s1=dff-fcil\&s2=farm-ferme\&s3=db-el.

Charlton, G. L., V. Bouffard, J. Gibbons, E. Vasseur, D. B. Haley, D. Pellerin, J. Rushen, and A. M. de Passillé. 2016. Can automated measures of lying time help assess lameness and leg lesions on tiestall dairy farms? Appl. Anim. Behav. Sci. 175:14-22.

Espejo, L. A., M. I. Endres, and J. A. Salfer. 2006. Prevalence of lameness in high-producing Holstein cows housed in freestall barns in Minnesota. J. Dairy Sci. 89:3052-3058. https://doi.org/10.3168/ jds.S0022-0302(06)72579-6.

Flower, F. C., and D. M. Weary. 2009. Locomotion assessment in dairy cattle. Animal 3:87-95.

Gibbons, J., D. B. Haley, J. H. Cutler, C. Nash, J. Z. Heyerhoff, D. Pellerin, S. Adam, A. Fournier, A. M. de Passillé, J. Rushen, and E. Vasseur. 2014. Technical note: A comparison of 2 methods of assessing lameness prevalence in tiestall herds. J. Dairy Sci. 97:350-353.

Higginson Cutler, J. H., J. Rushen, A. M. de Passillé, J. Gibbons, K. Orsel, E. Pajor, H. Barkema, L. Solano, D. Pellerin, D. Haley, and E. Vasseur. 2015. Producer estimations and perceived importance of lameness in dairy cattle: Where do we move from here? Page 45 in Proc. 49th International ISAE Congress, Sapporo, Hokkaido, Japan. 
Leach, K. A., S. Dippel, J. Huber, S. March, C. Winckler, and H. R. Whay. 2009. Assessing lameness in cows kept in tie-stalls. J. Dairy Sci. 92:1567-1574.

Rushen, J., A. Butterworth, and J. C. Swanson. 2011. Animal behavior and well-being symposium: Farm animal welfare assurance: Science and application. J. Anim. Sci. 89:1219-1228.

Solano, L., H. W. Barkema, E. A. Pajor, S. Mason, S. J. LeBlanc, J. C. Zaffino Heyerhoff, C. G. R. Nash, D. B. Haley, D. Pellerin, J. Rushen, A. M. de Passille, E. Vasseur, and K. Orsel. 2015. Prevalence of lameness and associated risk factors in Canadian HolsteinFriesian cows housed in freestall barns. J. Dairy Sci. 98:6978-6991.

USDA. 2010. Animal and Plant Health Inspection Services 2010. Facility characteristics and cow comfort on U.S. dairy operations, 2007. Accessed Jul. 3, 2016. https://www.aphis.usda.gov/animal_ health/nahms/dairy/downloads/dairy07/Dairy07_ir_Facilities. pdf.
Vasseur, E., J. Gibbons, J. Rushen, D. Pellerin, E. Pajor, D. Lefebvre, and A. M. de Passillé. 2015. An assessment tool to help producers improve cow comfort on their farms. J. Dairy Sci. 98:698-708.

Vasseur, E., J. Rushen, D. B. Haley, and A. M. de Passille. 2012 Sampling cows to assess lying time for on-farm animal welfare assessment. J. Dairy Sci. 95:4968-4977. https://doi.org/10.3168/ jds.2011-5176.

Westin, R., A. Vaughan, A. M. de Passille, T. J. DeVries, E. A. Pajor, D. Pellerin, J. M. Siegford, A. Witafi, E. Vasseur, and J. Rushen. 2016. Cow and farm-level risk factors for lameness on dairy farms with automated milking systems. J. Dairy Sci. 99:3732-3743.

Whay, H. R., D. C. J. Main, L. E. Green, and A. J. F. Webster. 2003. Assessment of the welfare of dairy cattle using animal-based measurements: Direct observations and investigation of farm records. Vet. Rec. 153:197-202 